\title{
Parametric contrast-enhanced ultrasound as an early predictor of radiation-based therapeutic response for lymph node metastases of nasopharyngeal carcinoma
}

\author{
ZHIMIN YE ${ }^{1}$, PINTONG HUANG ${ }^{2}$, XIAOFENG ZHOU ${ }^{1}$, QIAN HUANG $^{3}$, QIONGGE HU $^{1}$, \\ YONGJIE SHUI ${ }^{1}$, LI SHEN ${ }^{1}$, ENYIN LAI $^{3}$ and QICHUN WEI ${ }^{1,4}$
}

\begin{abstract}
Departments of ${ }^{1}$ Radiation Oncology, and ${ }^{2}$ Ultrasound, The Second Affiliated Hospital, Zhejiang University School of Medicine, Hangzhou 310009; ${ }^{3}$ Department of Physiology, Zhejiang University School of Medicine, Hangzhou 310058; ${ }^{4}$ National Ministry of Education Key Laboratory of Cancer Prevention and Intervention, Zhejiang University School of Medicine, Hangzhou 310009, P.R. China
\end{abstract}

Received March 26, 2014; Accepted April 15, 2014

DOI: $10.3892 / \mathrm{mco} .2014 .331$

\begin{abstract}
Nasopharyngeal carcinoma (NPC) is a common type of cancer in South East Asia with peculiar epidemiology, pathology, clinical behavior and response to treatment characteristics. To the best of our knowledge, this is the first study to investigate the use of a contrast-enhanced ultrasound (CEUS) as a predictor for the therapeutic response in lymph node metastases of NPC patients treated with radiation-based therapy. Sixty-seven NPC patients with lymph node metastases underwent the lymph nodes CEUS examination twice; pre- and in-treatment (at the 5th fraction radiotherapy), respectively. The CEUS parameters were acquired through Qontrast_4.0 software and mainly included peak intensity (PI) and time to peak (TTP). The response assessment at the lymph nodes revealed a complete response (CR) in 48 patients and partial response (PR) in 19 patients. There was a significant difference in pre-treatment $\mathrm{PI}\left(\mathrm{PI}_{\text {pre }}\right)$ between the patients who showed $\mathrm{CR}$ or PR, but the predicted sensitivity and specificity of $\mathrm{PI}_{\text {pre }}$ was low. The mean in-treatment PI $\left(\mathrm{PI}_{\mathrm{in}}\right)$ value of the lymph nodes that achieved a CR was $34.24 \pm 3.78 \%$, which was significantly higher than the $\mathrm{PI}_{\text {in }}$ value for $\mathrm{PR}, 25.62 \pm 2.30 \%(\mathrm{P}<0.001)$. Furthermore, the $\mathrm{PI}_{\text {ratio }}$, a PI-quotient, was calculated by dividing the $\mathrm{PI}_{\text {in }}$ by the corresponding $\mathrm{PI}_{\text {pre }}$. The higher $\mathrm{PI}_{\text {ratio }}$
\end{abstract}

Correspondence to: $\mathrm{Dr} \mathrm{Li}$ Shen, Department of Radiation Oncology, The Second Affiliated Hospital, Zhejiang University School of Medicine, 88 Jiefang Road, Hangzhou 310009, P.R. China E-mail: lilly0506@hotmail.com

Dr Qichun Wei, National Ministry of Education Key Laboratory of Cancer Prevention and Intervention, Zhejiang University School of Medicine, 88 Jiefang Road, Hangzhou 310009, P.R. China E-mail: Qichun_Wei@zju.edu.cn

Key words: contrast-enhanced ultrasound, nasopharyngeal carcinoma, lymph node metastasis, therapeutic response, parametric imaging was also observed in CR lymph nodes $(0.81 \pm 0.01$ vs. $0.66 \pm 0.01$; $\mathrm{P}=0.001)$, and the mean change in $\mathrm{PI}\left(\mathrm{PI}_{\Delta} ; \mathrm{PI}_{\Delta}=\mathrm{PI}_{\text {pre }}-\mathrm{PI}_{\text {in }}\right)$ was smaller in the patients with $\mathrm{CR}$ nodes compared to the patients with PR nodes (7.79 \pm 3.28 vs. $13.77 \pm 1.90 \%$; $\mathrm{P}=0.000)$. No difference was observed in $\mathrm{TTP}_{\text {pre }}$ or $\mathrm{TTP}_{\text {in }}$ between the $\mathrm{CR}$ or PR lymph nodes patients. A receiver operating characteristic curve was constructed to assess the accuracy of the parameters for the prediction of the therapeutic responses. The sensitivity and specificity of $\mathrm{PI}_{\text {in }}$ in predicting the therapeutic response was 94.3 and $88.2 \%$, and the corresponding figures of the $\mathrm{PI}_{\text {ratio }}$ were 92.5 and $83.8 \%$, respectively. The CEUS parameters during the early course of radiation-based therapy, $\mathrm{PI}_{\text {in }}$ and $\mathrm{PI}_{\text {ratio }}$, are associated with the therapeutic response of NPC lymph node metastases, with a high predicted sensitivity and specificity, thus yielding the conceivable predictors with the potential to individualize treatment.

\section{Introduction}

Nasopharyngeal carcinoma (NPC) occurs commonly in the Asian population, particularly in Southern and Southeast China (1). Due to the peculiar characteristics in its epidemiology, pathology, clinical behavior and response to treatment, NPC is different from other head and neck squamous cell cancer and has a relatively high overall survival rate with the integration of chemotherapy into radiotherapy $(2,3)$. However, the majority of NPC patients present with a late stage disease accompanying neck nodal metastases when diagnosed, and the cure rate for those advanced NPCs remains unsatisfactory (4). In addition, numerous NPC survivors are often affected by moderate to severe late complications, resulting from the impact of radiation on the organs that are adjacent to the nasopharynx and neck nodes, and chemotherapy in advanced cases further exacerbates these side-effects (5). Therefore, exploring novel therapeutic regimens and improvements in disease monitoring is required. If the therapeutic effect can be predicted prior to or at the early course of the treatment, it is possible to modify the therapeutic strategies for the remaining treatment. Novel therapeutic alternatives, 
such as anti-epidermal growth factor receptor monoclonal antibodies, including Cetuximab and Nimotuzumab, could be advised as a combination for patients likely to be resistant to conventional treatment.

Functional images, mainly including dynamic contrast-enhanced-computed tomography (DCE-CT), positron emission tomography (PET-CT), magnetic resonance spectrometry, diffusion-weighted images (DWI) and dynamic contrast-enhanced-magnetic resonance imaging (DCEMRI), play an important role in assessing the treatment effect on the solid tumor. Considering the apparent diffusion coefficient value of DWI and the perfusion parameter, $\mathrm{K}^{\text {trans }}$, of DCE-MRI as examples, the aforementioned parameters may allow the possibility to predict an early treatment response and prognosis for chemotherapy and radiotherapy $(6,7)$. The aforementioned image modalities are mainly dependent on contrast agents that may cause an allergic reaction. DCE-CT and PET use ionizing radiation, which has known risks. Contrast-enhanced ultrasound (CEUS) is another type of functional image. The medium of CEUS is SonoVue, which contains micrometer-sized (1-10 $\mu \mathrm{m}$ in diameter) bubbles of sulfur hexafluoride with a stabilizing shell. The introduction of exogenous microbubbles into the vasculature causes enhancement of the backscattered intensity of the blood and can be used to assess tissue blood flow. Relevant analysis software has been developed to analyze ultrasound signal intensity (SI) patterns obtained by imaging continuously prior or subsequent to treatment, and information regarding tissue blood flow and vascular integrity, branching patterns and density will be assessed (8). As reported previously, the information extracted from the CEUS data was similar to that obtained from DCE-MRI (9), and CEUS has numerous benefits, including a sufficient high safety profile that is acceptable for patients with renal failure or an iodine allergy, absence of radiation, easy reproducibility and high temporal resolution $(10-13)$.

Thus, the present study was performed to evaluate the potential utility of CEUS-derived parameters from the metastatic cervical lymph nodes, prior or subsequent to the early course of the treatment, in predicting the nodal treatment response to radiation-based therapy in patients with NPC.

\section{Patients and methods}

Patient selection. Sixty-seven NPC with cervical lymph node metastases patients who were treated at The Second Affiliated Hospital (Zhejiang University School of Medicine, Hangzhou, China) between December 2011 and February 2013, were enrolled in the study. The study was approved by the Institutional review board, and written informed consent was obtained from each participant prior to the CEUS examination. All the subjects qualified for the following criteria: i) NPC with metastatic lymph nodes proven by pathology; ii) Eastern Cooperative Oncology Group performance status score, $\leq 2$; iii) adequate organ function; and iv) no concomitant malignancy. The stage of disease was classified according to the 7 th edition of the Union for International Cancer Control (UICC) and the American Joint Committee on Cancer (AJCC) staging system (14). All the clinical characteristics of the patient are listed in Table I.
Treatment protocol. The first CEUS examinations were performed prior to any treatment, and the second CEUS examinations were arranged at the 5th fraction of radiotherapy (using the CEUS methodology). All the 67 patients underwent intensity-modulated radiotherapy (IMRT) and among them, 52 patients received platinum-based concomitant chemotherapy and 10 received weekly Nimotuzumab-targeted therapy at a dose of $200 \mathrm{mg} 1$ week before and during the course of radiotherapy and the other 5 cases received only IMRT. Nimotuzumab, a monoclonal antibody against epidermal growth factor receptor, has been officially approved by the State Food and Drug Administration of China to treat advanced NPC (15). The target volume contouring was made following the guideline of the Radiation Therapy Oncology Group Contouring Atlas (http://www.rtog.org/ CoreLab/ContouringAtlases/HNAtlases.aspx). The planning target volume of the metastatic lymph nodes was defined as $\mathrm{PCTV}_{\text {nd }}$. IMRT was performed using 6-MV photon beams and the IMRT plan was normalized such that $95 \%$ of the PCTV was covered with the prescription dose [60-70 Gy/30-32 fractions], and all the patients underwent IMRT once daily and 5 fractions a week.

CEUS methodology. The first section focused exclusively on the pre-treatment CEUS examination. All the ultrasound investigations were performed using the Sequoia 512 Acuson sonographic system (Siemens Healthcare, Erlangen, German) equipped with Cadence ${ }^{\mathrm{TM}}$ contrast pulse-sequencing visualization technology and a high-resolution broadband ultrasound transducer (8L5; 5-8 MHz). Each dose of the intravenous contrast medium of microbubbles (SonoVue; Bracco, Milan, Italy) was dissolved in $5 \mathrm{ml}$ of saline and a $2.4 \mathrm{ml}$ bolus was injected into the superficial elbow vein of the patient at the rate of $1 \mathrm{ml} / \mathrm{sec}$, followed by a $5.0 \mathrm{ml}$ saline flush (16). The process of CEUS was performed by the same ultrasound investigator with $>5$ years of experience in CEUS. The process of CEUS should include the following: i) Scan parameters (depth, focus, pulse repletion frequency, mechanical index and depth-gain compensation) were optimized for a clear, artifact-free depiction; ii) the probe was manually stabilized at the largest diameter of the target lymph node; and iii) the duration of the video was $290 \mathrm{sec}$ for analysis (17). The video was stored as a digital archive (Audio Video Interleave) in the hard disc and was transferred to a personal computer for off-line parametric analysis.

The second section was in-treatment CEUS examination, and each of the 67 patients underwent the aforementioned CEUS examination at the 5 th fraction radiotherapy once again. To assure agreement of the lymph nodes examination with the first examination, the ultrasound investigator reviewed the previous CEUS video and subsequently performed the second examination. All the patients underwent the CEUS examinations twice; the former digital archive was the baseline as a control, whereas the later digital archive was under the treatment as a comparison.

Off-line parametric CEUS analysis. The aforementioned digital archives were processed with the use of contrast-enhanced computer-assisted perfusion analysis of the metastatic nodes using the Qontrast ${ }^{\mathrm{TM}}$ analysis software (Qontrast 4.0; Bracco SpA, Milan, Italy), which is a 
post-processing computational tool and can be used to obtain objective and quantitative parameters of the microvessels in various organs, including the lymph node (6). A region of interest (ROI) encompassing the whole area of the lymph node was manually drawn, and subsequently the software automatically processed and a time-intensity curve and parametric graphs were produced. The following parameters were automatically generated (Fig. 1): i) Peak intensity [PI; including pre-treatment $\left(\mathrm{PI}_{\text {pre }}\right)$ and in-treatment $\left(\mathrm{PI}_{i n}\right)$ as percentages] defined as the increase in signal intensity (SI) from baseline SI to the maximal SI measured in the selected ROI; and ii) time to peak (TTP; including $\mathrm{TTP}_{\text {pre }}$ and $\mathrm{TTP}_{\text {in }}$, in sec) defined as the time period from the onset of the lymph node enhancement to the moment the maximal SI is reached. The aforementioned analyses were all performed by the same investigator who was well experienced in the Qontrast software, and was blinded to the clinical data. To maintain intra-investigator agreement, $>3$ repeats of the aforementioned analysis were carried out by the same investigator. Concordant measurements were those that differed by no more than \pm 1 sec. Subsequently, $\mathrm{PI}_{\Delta}$ and $\mathrm{PI}_{\text {ratio }}$ were calculated by the algorithm that represented the change of the contrast agent perfusion via treatment: $\mathrm{PI}_{\Delta}=\mathrm{PI}_{\mathrm{pre}}-\mathrm{PI}_{\mathrm{in}}$ and $\mathrm{PI}_{\text {ratio }}=\mathrm{PI}_{\text {in }} / \mathrm{PI}_{\text {pre }}$.

Notably, the Qontrast software can compensate for minor changes in the imaging plane (such as from extremely shallow breathing). In the case of more pronounced changes in the imaging plane, frame-by-frame editing can be performed, and the respective frames can be manually selected and characterized as 'wrong' (18). By contrast, Qontrast-assisted CEUS parameters exhibited high inter-investigator reproducibility (11).

Evaluation of the therapeutic response. CE-MRI was recommended as the tool for evaluating the treatment effect of the metastatic cervical lymph nodes. Each patient underwent the CE-MRI examination twice, the former arranged prior to any treatment and the latter scheduled 1 month after the completion of all the radiation fractions. MR images were interpreted by the same experienced radiation oncologist with $>10$ years of clinical experience in NPC, who was informed of the target lymph nodes in advance. The Response Evaluation Criteria in Solid Tumors (RECIST) 1.1 criteria was referred to for assessment of the therapeutic response (19).

Statistical analysis. All the statistical analyses were carried out using SPSS 16.0 software (SPSS, Inc., Chicago, IL, USA). The descriptive statistics were produced for the continuous variables and the results are presented as mean \pm standard deviation (SD). The $\chi^{2}$ or Fisher's exact tests were used to determine the significance of the associations between the therapeutic effect and categorical variables, whereas the correlation between the continuous variables and the therapeutic effect was assessed by the analysis of variance. The statistical significance of the changes in PI and TTP was evaluated with the paired t-test. All the tests were two-sided and $\mathrm{P}<0.05$ was considered to indicate a statistical significance difference. The Spearman's correlation coefficient between the situation of the lymph nodes and changes in the perfusion parameters was calculated. The correlations were interpreted according to Cohen's standard, in which absolute correlations of $<0.3$ were considered weak, 0.3-0.5 were moderate and $0.5-1.0$ were strong. A receiver-operating
Table I. Clinical data according to the therapeutic response for nasopharyngeal carcinoma patients with lymph node metastases, $\mathrm{n}=67$.

\begin{tabular}{|c|c|c|c|}
\hline \multirow[b]{2}{*}{ Characteristics } & \multicolumn{2}{|c|}{ Therapeutic response } & \multirow[b]{2}{*}{ P-value } \\
\hline & CR & PR & \\
\hline Patients, $\mathrm{n}$ & 48 & 19 & \\
\hline Age, years & $55.83 \pm 10.38$ & $56.08 \pm 10.94$ & 0.912 \\
\hline Gender, $\mathrm{n}$ & & & 0.782 \\
\hline Male & 26 & 11 & \\
\hline Female & 22 & 8 & \\
\hline $\mathrm{PS}, \mathrm{n}$ & & & 0.492 \\
\hline 0 & 39 & 14 & \\
\hline 1 & 9 & 5 & \\
\hline Treatment, $\mathrm{n}$ & & & 0.023 \\
\hline RT & 6 & 7 & \\
\hline $\mathrm{RT}+\mathrm{CT} / \mathrm{T}$ & 42 & 12 & \\
\hline Differentiation, $\mathrm{n}$ & & & 0.310 \\
\hline Well/moderate & 4 & 2 & \\
\hline Poor & 44 & 17 & \\
\hline T stage, $\mathrm{n}$ & & & 0.686 \\
\hline $\mathrm{T} 1$ & 5 & 3 & \\
\hline $\mathrm{T} 2$ & 28 & 9 & \\
\hline T3 & 9 & 6 & \\
\hline $\mathrm{T} 4$ & 6 & 1 & \\
\hline $\mathrm{N}$ stage, $\mathrm{n}$ & & & 0.021 \\
\hline N1 & 27 & 4 & \\
\hline $\mathrm{N} 2$ & 19 & 12 & \\
\hline N3 & 2 & 3 & \\
\hline $\begin{array}{l}\text { Lymph node } \\
\text { Max. D, cm }\end{array}$ & $3.24 \pm 0.92$ & $3.83 \pm 0.93$ & 0.005 \\
\hline
\end{tabular}

CR, complete response; PR, partial response; PS, performance status; RT, radiotherapy; CT, chemotherapy; T, targeted therapy; Max. D, maximum diameter.

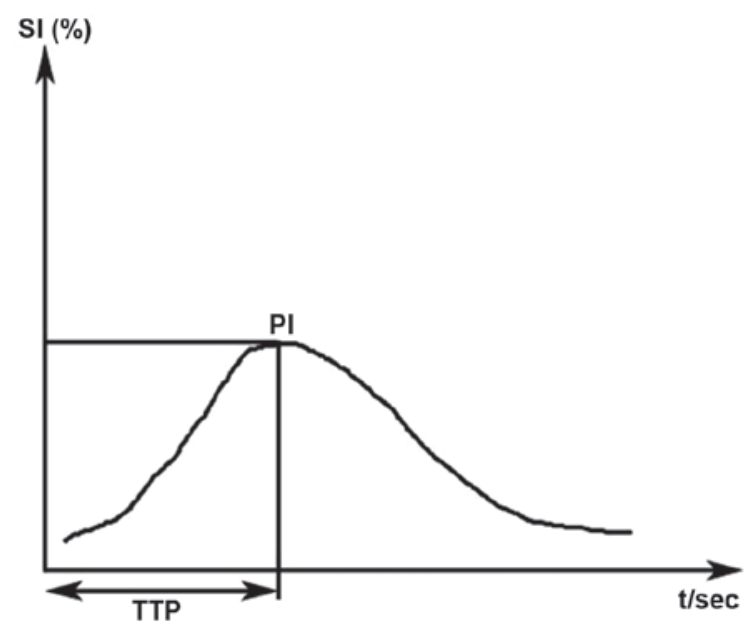

Figure 1. Schematic diagram showing the parameters extrapolated by the Qontrast 4.0 analysis software of the time-intensity curve in the lymph node. SI, signal intensity; PI, peak intensity; TTP, time to peak. 
Table II. CEUS parameters with different therapeutic responses for lymph node metastases of nasopharyngeal carcinoma patients, $n=67$.

\begin{tabular}{lrrr}
\hline Parameters & $\mathrm{CR}(\mathrm{n}=48)$ & $\mathrm{PR}(\mathrm{n}=19)$ & P-value \\
\hline $\mathrm{PI}_{\text {pre }}, \%$ & $41.90 \pm 3.62$ & $39.39 \pm 2.48$ & 0.002 \\
$\mathrm{PI}_{\text {in }}, \%$ & $34.24 \pm 3.78$ & $25.62 \pm 2.30$ & 0.001 \\
$\mathrm{TTP}_{\text {pre }}$, sec & $10.92 \pm 0.26$ & $11.07 \pm 0.61$ & 0.334 \\
$\mathrm{TTP}_{\text {in }}$, sec & $12.42 \pm 1.49$ & $12.13 \pm 1.40$ & 0.356 \\
$\mathrm{PI}_{\Delta}, \%$ & $7.79 \pm 3.28$ & $13.77 \pm 1.90$ & 0.001 \\
$\mathrm{PI}_{\text {ratio }}$ & $0.81 \pm 0.01$ & $0.66 \pm 0.01$ & 0.001 \\
\hline
\end{tabular}

CEUS, contrast-enhanced ultrasound; $\mathrm{CR}$, complete response; PR, partial response; PI, peak intensity; pre, pre-treatment; in, in-treatment.

A

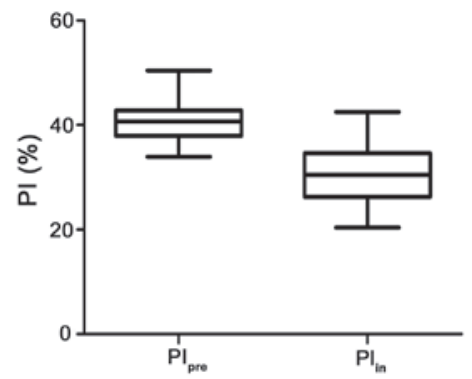

B

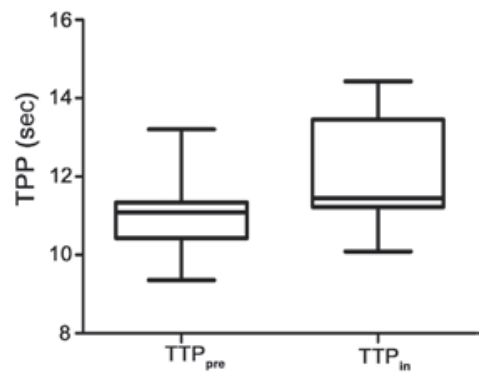

Figure 2. Box and whisker plots showing the statistically significant changes observed in (A) peak intensity (PI) and (B) time to peak (TTP) (the upper and lower end of each box corresponds to the maximum and minimum value, respectively, and the horizontal line inside the box corresponds to the mean value).

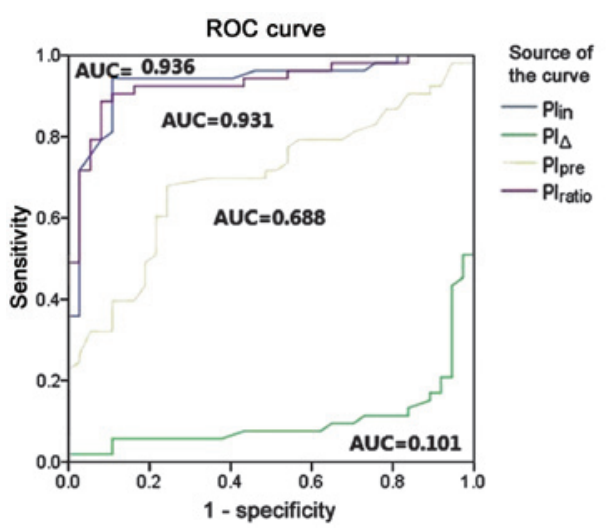

Figure 3. Receiver operating characteristic (ROC) curve constructed to depict predictive probabilities of the logistic regression model for the imaging parameters obtained [peak intensity $(\mathrm{PI})_{\text {in }}$ and $\mathrm{PI}_{\text {ratio }}$ are significant predictors compared to $\mathrm{PI}_{\text {pre }}$ and $\left.\mathrm{PI}_{\Delta}\right]$. AUC, area under curve. characteristic (ROC) curve was constructed to assess the accuracy of the parameters for the prediction of the therapeutic responses. The ROC was constructed to illustrate the predicted probability of the parameters.

\section{Results}

Clinical data and treatment response. Of the 67 patients, there were 37 males and 30 females, with a mean age of $55.93 \pm 10.55$ years (range, $24-88$ years). The baseline patient and tumor characteristics are summarized in Table I. All the patients completed the entire radiation-therapy plan. The response assessment at the lymph nodes revealed a complete response (CR) in 48 patients and partial response (PR) in 19 patients. No patients showed stable or progressive disease at the lymph nodes or at the primary site. Age, gender, performance status score, T stage or pathological differentiation status were not associated with the treatment response $(\mathrm{P}>0.05)$. However, $\mathrm{N}$ stage, the size of the lymph node and the treatment modalities were significantly different between the $\mathrm{CR}$ and $\mathrm{PR}$ groups, respectively $(\mathrm{P}<0.05)$. Notably, radiation therapy in combination with chemotherapy/targeted therapy was superior to radiotherapy alone, in regards to therapeutic response (Table I).

Data of CEUS parameters and therapeutic response. For all the 67 cases investigated, $\mathrm{PI}_{\text {pre }}$ ranged from 33.9-50.4\% $(40.9 \pm 3.4 \%)$, and the $\mathrm{PI}$ following 5 fractions of radiation $\left(\mathrm{PI}_{\mathrm{in}}\right)$ ranged from $20.4-42.5 \%(30.7 \pm 5.3 \%)$. There was a significant difference in $\mathrm{PI}_{\text {pre }}$ between the patients who showed a CR or PR of the metastatic lymph nodes, and the mean values of $\mathrm{PI}_{\text {pre }}$ were higher in patients with $\mathrm{CR}$ nodes compared to the patients with PR nodes $(41.90 \pm 3.62$ vs. $39.39 \pm 2.48 \%$; $\mathrm{P}=0.002$ ). Following 5 fractions of radiation, a decrement in PI was observed in all the patients without any exception (Fig. 2A). The mean $\mathrm{PI}_{\text {in }}$ value of the lymph nodes that achieved CR was $34.24 \pm 3.78 \%$, which was significantly higher compared to the $\mathrm{PI}_{\text {in }}$ value for the PR, 25.62 $\pm 2.30 \%(\mathrm{P}<0.001)$. To further standardize the data, a PI-quotient known as $\mathrm{PI}_{\text {ratio }}$, was calculated by dividing the $\mathrm{PI}_{\text {in }}$ by the corresponding $\mathrm{PI}_{\text {pre }}$ for the same lymph node. A higher $\mathrm{PI}_{\text {ratio }}$ was also observed in the CR lymph nodes $(0.81 \pm 0.01$ vs. $0.66 \pm 0.01 ; \mathrm{P}=0.001)$. As shown in Table II, the mean change in $\mathrm{PI}\left(\mathrm{PI}_{\Delta} ; \mathrm{PI}_{\Delta}=\mathrm{PI}_{\text {pre }}-\mathrm{PI}_{\text {in }}\right)$ was smaller in the patients with $\mathrm{CR}$ nodes compared to the patients with PR nodes $(7.79 \pm 3.28$ vs. $13.77 \pm 1.90 \%$; $\mathrm{P}<0.001)$.

In the present study, $\mathrm{TTP}_{\text {pre }}$ ranged from 9.36 to $13.21 \mathrm{sec}$ $(10.98 \pm 0.69 \mathrm{sec})$, and $\mathrm{TTP}_{\text {in }}$ ranged from 10.09 to $14.43 \mathrm{sec}$ $(12.30 \pm 1.46 \mathrm{sec})$, showing that the value of TTP had a tendency to increase following treatment and the change in TTP was statistically significant $(\mathrm{P}<0.05)$ (Fig. 2B). However, the mean $\mathrm{TTP}_{\text {pre }}$ was $10.92 \pm 0.26 \mathrm{sec}$ in the $\mathrm{CR}$ nodes and $11.07 \pm 0.61 \mathrm{sec}$ in the PR nodes. No significant difference was observed between the two groups $(\mathrm{P}=0.334)$. Similarly, there was no significant difference in $\mathrm{TTP}_{\text {in }}$ between patients who showed a CR or PR of the metastatic lymph nodes $(12.42 \pm 1.49$ vs. $12.13 \pm 1.40 ; \mathrm{P}=0.356)$, as shown in Table II.

Correlation between the CEUS parameters and therapeutic response. The Spearman's correlation coefficient between the lymph nodes therapeutic response and changes in CEUS perfusion parameters was calculated. There was a strong-positive 
Table III. Spearman's rank correlation coefficients for the CEUS parameters and therapeutic response.

\begin{tabular}{|c|c|c|c|c|c|c|c|}
\hline & Therapeutic response & $\mathrm{PI}_{\mathrm{pre}}$ & $\mathrm{PI}_{\mathrm{in}}$ & $\mathrm{TTP}_{\text {pre }}$ & $\mathrm{TTP}_{\text {in }}$ & $\mathrm{PI}_{\Delta}$ & $\mathrm{PI}_{\text {ratio }}$ \\
\hline $\begin{array}{l}\text { Therapeutic } \\
\text { response }\end{array}$ & 1 & & & & & & \\
\hline $\mathrm{PI}_{\mathrm{pre}}$ & $0.368^{\mathrm{a}}$ & 1 & & & & & \\
\hline $\mathrm{PI}_{\mathrm{in}}$ & $0.810^{\mathrm{a}}$ & $0.681^{\mathrm{a}}$ & 1 & & & & \\
\hline TTP $_{\text {pre }}$ & -0.141 & -0.159 & -0.156 & 1 & & & \\
\hline $\operatorname{TTP}_{\text {in }}$ & 0.121 & 0.009 & 0.129 & 0.164 & 1 & & \\
\hline PI & $-0.777^{\mathrm{a}}$ & 0.120 & $-0.785^{\mathrm{a}}$ & 0.066 & -0.188 & 1 & \\
\hline $\mathrm{PI}_{\text {ratio }}$ & $0.734^{\mathrm{b}}$ & $-0.263^{\mathrm{a}}$ & $0.868^{\mathrm{b}}$ & 0.070 & -0.204 & $-0.956^{\mathrm{b}}$ & 1 \\
\hline
\end{tabular}

${ }^{\mathrm{a}} \mathrm{P}<0.05 ;{ }^{\mathrm{b}} \mathrm{P}<0.01$. CEUS, contrast-enhanced ultrasound; PI, peak intensity; pre, pre-treatment; in, in-treatment.

correlation between the $\mathrm{PI}_{\mathrm{in}}, \mathrm{PI}_{\text {ratio }}$ and therapeutic response $(\varrho=0.81, \varrho=0.734)$, a moderate-positive correlation between the $\mathrm{PI}_{\text {pre }}$ and therapeutic response $(\mathrm{Q}=0.368)$ and a strong-negative correlation between the therapeutic response and $\mathrm{PI}_{\Delta}(\mathrm{Q}=-0.777)$ (Table III). Logistic regression analysis of the CEUS parameters indicated that $\mathrm{PI}_{\text {in }}$ and $\mathrm{PI}_{\text {ratio }}$ were the significant predictors of the therapeutic response $(\mathrm{P}<0.01)$.

An ROC curve was constructed to assess the accuracy of the parameters for the prediction of the therapeutic responses. The ROC curve showed that the therapeutic response could be well predicted by the parameters of $\mathrm{PI}_{\text {in }}$ and $\mathrm{PI}_{\text {ratio }}$ compared to $\mathrm{PI}_{\text {pre }}$ and $\mathrm{PI}_{\Delta}$. The $\mathrm{PI}_{\text {in }}$ area under the ROC curve (AUC) was 0.936 (95\% confidence interval, 0.877-0.988), and the AUC of the $\mathrm{PI}_{\text {ratio }}$ was 0.931 (95\% confidence interval, 0.877-0.985) (Fig. 3). When the cut-off value of $\mathrm{PI}_{\text {in }}$ was set at $29.4 \%$, sensitivity and specificity in predicting the lymph node therapeutic response was 94.3 and $88.2 \%$. The best cut-off value of the $\mathrm{PI}_{\text {ratio }}$ was $\geq 0.69$ by coordinating the points of the ROC curve, and the predicted sensitivity and specificity of the $\mathrm{PI}_{\text {ratio }}$ was 92.5 and $83.8 \%$, respectively (Table IV).

\section{Discussion}

To the best of our knowledge, the present study is the first to assess the early predictive value of parametric CEUS for the therapeutic response in the metastatic cervical lymph nodes of NPC patients treated with radiation-based therapy. The aim was to evaluate whether any of these CEUS parameters are suitable as a predictor for the nodal treatment response to radiation-based therapy in NPC patients with cervical lymph nodes metastases.

In the present study, a higher mean value of $\mathrm{PI}_{\text {pre }}$ was observed in the lymph nodes that achieved CR compared to $\mathrm{PR}$, and the difference was statistically significant. Functional images, including DCE-MRI, have been employed for the prediction of the early treatment response and prognosis for head and neck cancers $(18,20,21)$. DCE-MRI provides a perfusion parameter, $\mathrm{K}^{\text {trans }}$, which reflects a combination of the tumor blood flow and microvascular permeability. In the study reported by Chawla et al (18), patients with head and neck squamous cell carcinoma that was responsive to chemoradiation therapy had significantly higher pre-treatment $\mathrm{K}^{\text {trans }}$ values from nodal masses than patients with a PR. In another
Table IV. Sensitivity and specificity for the CEUS parameters, $\mathrm{PI}_{\text {pre }}, \mathrm{PI}_{\text {in }}$ and $\mathrm{PI}_{\text {ratio. }}$.

Parameters Cut-off value Sensitivity, $\% \quad$ Specificity, $\%$

\begin{tabular}{llll}
\hline $\mathrm{PI}_{\text {pre }}$ & $\geq 39.65 \%$ & 72.0 & 52.0 \\
$\mathrm{PI}_{\text {in }}$ & $\geq 29.40 \%$ & 94.3 & 88.2 \\
$\mathrm{PI}_{\text {ratio }}$ & $\geq 0.69$ & 92.5 & 83.8 \\
\hline
\end{tabular}

CEUS, contrast-enhanced ultrasound; PI, peak intensity; pre, pre-treatment; in, in-treatment.

cohort of 33 patients with head and neck squamous cell carcinoma who were treated with chemoradiotherapy, the average pre-treatment $\mathrm{K}^{\text {trans }}$ value of the $\mathrm{CR}$ group was found to be significantly higher $(\mathrm{P}=0.001)$ than that of the $\mathrm{PR}$ group (21). The result of the present study is partially consistent with the aforementioned DCE-MRI studies. However, the majority of the $\mathrm{PI}_{\text {pre }}$ values fell in a wide region and there was a large range of $\mathrm{PI}_{\text {pre }}$ values from the $\mathrm{CR}$ and $\mathrm{PR}$ groups that overlapped with each other. The predicted sensitivity and specificity of $\mathrm{PI}_{\text {pre }}$ was relatively low, and therefore the $\mathrm{PI}_{\text {pre }}$ value alone is of less interest for predicting the therapeutic response of the lymph node metastases from NPC.

We hypothesized that alternations in the metastatic nodal perfusion parameters during the early course of treatment for NPC may improve the predictive value for the therapeutic response than pre-treatment measurements alone. Thus, the second CEUS examinations were arranged at the 5 th fraction of radiotherapy for all the 67 patients. A decrement in PI was observed in all the investigated nodes regarding the $\mathrm{PI}_{\text {pre }}$. There was a significant difference in the $\mathrm{PI}_{\text {in }}$ between the patients who showed $\mathrm{CR}$ or $\mathrm{PR}$, and the values of $\mathrm{PI}_{\text {in }}$ were much higher in the patients with CR nodes $(34.24 \pm 3.78 \%)$ compared to those with PR nodes $(25.62 \pm 2.30 \%)$. For each individual lymph node, the $\mathrm{PI}_{\text {ratio }}$ was calculated by dividing the $\mathrm{PI}_{\text {in }}$ by the $\mathrm{PI}_{\text {pre }}$, and a higher $\mathrm{PI}_{\text {ratio }}$ was also observed in the lymph nodes that achieved a CR $(0.81 \pm 0.01$ vs. $0.66 \pm 0.01$; $\mathrm{P}=0.001)$. The $\mathrm{PI}_{\Delta}$ was found to be smaller in the CR lymph node. These findings suggest that an improved blood supply and potentially improved oxygenation during the early 
course of treatment may be a positive indicator for the therapeutic response at the metastatic lymph node of NPC. Based on the ROC curve, a cut-off for the $\mathrm{PI}_{\text {in }}$ and $\mathrm{PI}_{\text {ratio }}$ were established, which predicted the response with a specificity of 88.2 and $83.8 \%$, and a sensitivity of 94.3 and $92.5 \%$, respectively. To the best of our knowledge, the present study describes for the first time the CEUS parameters during the early course of chemo-radiotherapy that reliably predict the metastatic lymph node response. These parameters may help to optimize the patient selection, thereby individualizing treatment and preventing non-responders from undesirable side-effects.

TTP represents the arrival time of the contrast agent to reach its maximum. In the present study, TTP had a tendency to increase 1 week after the initiation of the radiation-based treatment. However, $\mathrm{TTP}_{\text {pre }}$ and $\mathrm{TTP}_{\text {in }}$ were found to have no significant difference regarding the $\mathrm{CR}$ or $\mathrm{PR}$ lymph node response status. In a study by Knieling et al (22), hepatocellular carcinoma patients were treated with sorafenib and the TTP increased as early as 1 month after the initiation of the treatment in the responder group compared to the non-responder group. In the study by Schirin-Sokhan et al (23), non-primary resectable liver metastases from colorectal cancer were treated with bevacizumab-based chemotherapy and it was demonstrated that the baseline TTP was significantly lower in the responder group compared to the non-responders, suggesting that low baseline TTP significantly correlates with tumor response according to RECIST. Furthermore, correlating to the antiangiogenic effect of bevacizumab, a strong increase in TTP was observed during chemotherapy, which was restricted to the responder group. The data concerning TTP and radiation-therapeutic response are sparse and should be investigated further in a larger patient population with more CEUS examinations during the course of the radiation-based therapy.

The patients treated with radiotherapy combined with chemotherapy and/or targeted therapy had a higher CR rate of lymph node metastases compared to those who received radiotherapy alone in the present study. A conventional radiotherapy course is usually 6-7 weeks, and may be followed by adjuvant chemotherapy and targeted therapy. If the early changes in nodal perfusion could help to predict the therapeutic response, it will be possible to alter the intensity of the treatment regimen, thus individualizing the remaining treatment.

The present study is, to the best of our knowledge, the first to investigate CEUS as a predictor for the therapeutic response in NPC cervical lymph nodes metastases. The data suggests that the CEUS parameters during the early course of chemo-radiotherapy, $\mathrm{PI}_{\text {in }}$ and $\mathrm{PI}_{\text {ratio }}$, are associated with the therapeutic response of the lymph node metastases from NPC, thus yielding conceivable predictors with the potential to modify and individualize treatment.

\section{Acknowledgements}

The authors would like to thank all the colleagues in the Department of Radiation Oncology and Ultrasound for their good cooperation. The present study was supported by the National Natural Science Foundation of China (grant no. 81071823 and 81201811) and the Zhejiang University Research Foundation.

\section{References}

1. Wei WI and Sham JS: Nasopharyngeal carcinoma. Lancet 365: 2041-2054, 2005

2. Razak AR, Siu LL, Liu FF, Ito E, O'Sullivan B and Chan K: Nasopharyngeal carcinoma: the next challenges. Eur J Cancer 46: 1967-1978, 2010.

3. Xiao WW, Huang SM, Han F, Wu SX, Lu LX, Lin CG, Deng XW, Lu TX, Cui NJ and Zhao C: Local control, survival, and late toxicities of locally advanced nasopharyngeal carcinoma treated by simultaneous modulated accelerated radiotherapy combined with cisplatin concurrent chemotherapy: long-term results of a phase 2 study. Cancer 117: 1874-1883, 2011.

4. Chan AT, Hsu MM, Goh BC, Hui EP, Liu TW, Millward MJ, Hong RL, Whang-Peng J, Ma BB, To KF, Mueser M, Amellal N, Lin X and Chang AY: Multicenter, phase II study of cetuximab in combination with carboplatin in patients with recurrent or metastatic nasopharyngeal carcinoma. J Clin Oncol 23: 3568-3576, 2005.

5. Lee AW, Tung SY, Chan AT, Chappell R, Fu YT, Lu TX, Tan T, Chua DT, O'sullivan B, Xu SL, Pang ES, Sze WM, Leung TW, Kwan WH, Chan PT, Liu XF, Tan EH, Sham JS, Siu L and Lau WH: Preliminary results of a randomized study (NPC-9902 Trial) on therapeutic gain by concurrent chemotherapy and/or accelerated fractionation for locally advanced nasopharyngeal carcinoma. Int J Radiat Oncol Biol Phys 66: 142-151, 2006.

6. Cao Y, Popovtzer A, Li D, Chepeha DB, Moyer JS, Prince ME, Worden F, Teknos T, Bradford C, Mukherji SK and Eisbruch A: Early prediction of outcome in advanced head-and-neck cancer based on tumor blood volume alterations during therapy: a prospective study. Int J Radiat Oncol Biol Phys 72: 1287-1290, 2008.

7. Korpanty G, Carbon JG, Grayburn PA, Fleming JB and Brekken RA: Monitoring response to anticancer therapy by targeting microbubbles to tumor vasculature. Clin Cancer Res 13: 323-330, 2007.

8. Chen JJ, Fu SY, Chiang CS, Hong JH and Yeh CK: A preclinical study to explore vasculature differences between primary and recurrent tumors using ultrasound Doppler imaging. Ultrasound Med Biol 39: 860-869, 2013.

9. Yankeelov TE, Niermann KJ, Huamani J, Kim DW, Quarles CC, Fleischer AC, Hallahan DE, Price RR and Gore JC: Correlation between estimates of tumor perfusion from microbubble contrast-enhanced sonography and dynamic contrast-enhanced magnetic resonance imaging. J Ultrasound Med 25: 487-497, 2006.

10. Beaton C, Cochlin D and Kumar N: Contrast enhanced ultrasound should be the initial radiological investigation to characterise focal liver lesions. Eur J Surg Oncol 36: 43-46, 2010.

11. Ridolfi F, Abbattista T, Busilacchi P and Brunelli E: Contrast-enhanced ultrasound evaluation of hepatic microvascular changes in liver diseases. World J Gastroenterol 18: 5225-5230, 2012.

12. Rissanen TT, Korpisalo P, Karvinen H, Liimatainen T, Laidinen S, Gröhn OH and Ylä-Herttuala S: High-resolution ultrasound perfusion imaging of therapeutic angiogenesis. JACC Cardiovasc Imaging 1: 83-91, 2008.

13. Badea AF, Tamas-Szora A, Clichici S, Socaciu M, Tăbăran AF, Băciut G, Cătoi C, Mureşan A, Buruian M and Badea R: Contrast enhanced ultrasonography (CEUS) in the characterization of tumor microcirculation. Validation of the procedure in the animal experimental model. Med Ultrason 15: 85-94, 2013.

14. Shah JP, Ang K, Baatenburg De Jong RJ, et al: Head and neck. In: AJCC Cancer Staging Manual. Edge SB, Byrd DR, Compton CC, Fritz AG, Greene FL and Trotti A (eds). 7th edition. Springer, New York, pp21-100, 2010.

15. Ramakrishnan MS, Eswaraiah A, Crombet T, Piedra P, Saurez G, Iyer H and Arvind AS: Nimotuzumab, a promising therapeutic monoclonal for treatment of tumors of epithelial origin. MAbs 1: 41-48, 2009

16. Ang J, Hu L, Huang PT, Wu JX, Huang LN, Cao CH, Zheng YX and Chen L: Contrast-enhanced ultrasonography assessment of gastric cancer response to neoadjuvant chemotherapy. World J Gastroenterol 18: 7026-7032, 2012.

17. Moschouris H, Malagari K, Marinis A, Kornezos I, Stamatiou K, Nikas G, Papadaki MG and Gkoutzios P: Hepatocellular carcinoma treated with transarterial chemoembolization: Evaluation with parametric contrast-enhanced ultrasonography. World J Radiol 4: 379-386, 2012. 
18. Chawla S, Kim S, Dougherty L, Wang S, Loevner LA, Quon H and Poptani H: Pretreatment diffusion-weighted and dynamic contrast-enhanced MRI for prediction of local treatment response in squamous cell carcinomas of the head and neck. AJR Am J Roentgenol 200: 35-43, 2013.

19. Eisenhauer EA, Therasse P, Bogaerts J, Schwartz LH, Sargent D, Ford R, Dancey J, Arbuck S, Gwyther S, Mooney M, Rubinstein L, Shankar L, Dodd L, Kaplan R, Lacombe D and Verweij J: New response evaluation criteria in solid tumours: revised RECIST guideline (version 1.1). Eur J Cancer 45: 228-247, 2009.

20. Shukla-Dave A, Lee NY, Jansen JF, Thaler HT, Stambuk HE, Fury MG, Patel SG, Moreira AL, Sherman E, Karimi S, Wang Y, Kraus D, Shah JP, Pfister DG and Koutcher JA: Dynamic contrast-enhanced magnetic resonance imaging as a predictor of outcome in head-and-neck squamous cell carcinoma patients with nodal metastases. Int J Radiat Oncol Biol Phys 82: 1837-1844, 2012.
21. Kim S, Loevner LA, Quon H, Kilger A, Sherman E, Weinstein G, Chalian A and Poptani H: Prediction of response to chemoradiation therapy in squamous cell carcinomas of the head and neck using dynamic contrast-enhanced MR imaging. AJNR Am J Neuroradiol 31: 262-268, 2010

22. Knieling F, Waldner MJ, GoertzRS,Zopf S, Wildner D, Neurath MF, Bernatik T and Strobel D: Early response to anti-tumoral treatment in hepatocellular carcinoma - can quantitative contrast-enhanced ultrasound predict outcome? Ultraschall Med 34: 38-46, 2013.

23. Schirin-Sokhan R, Winograd R, Roderburg C, Bubenzer J, do Ó NC, Guggenberger D, Hecker H, Trautwein $\mathrm{C}$ and Tischendorf JJ: Response evaluation of chemotherapy in metastatic colorectal cancer by contrast enhanced ultrasound. World J Gastroenterol 18: 541-545, 2012. 